\title{
Outcomes of Microendoscopic Discectomy and Percutaneous Transforaminal Endoscopic Discectomy for the Treatment of Lumbar Disc Herniation: A Comparative Retrospective Study
}

\author{
Arjun Sinkemani, Xin Hong, Zeng-Xin Gao, Su-Yang Zhuang, Zan-Li Jiang, Shao-Dong Zhang, \\ Jun-Ping Bao, Lei Zhu, Pei Zhang, Xin-Hui Xie, Feng Wang, Xiao-Tao Wu \\ Department of Spine Surgery, Zhongda Hospital, Medical School of Southeast University, Nanjing, China
}

\begin{abstract}
Study Design: Retrospective, case control evaluation of 86 patients who underwent microendoscopic discectomy (MED) and percutaneous transforaminal endoscopic discectomy (PTED) for the treatment of lumbar disc herniation (LDH).

Purpose: To evaluate the safety and the outcomes of MED and PTED for the treatment of LDH.

Overview of Literature: MED and PTED are minimally invasive surgical techniques for lower back pain. Studies to date have shown that MED and PTED are safe and effective treatment modalities for LDH.

Methods: A retrospective study was performed in patients with LDH treated with MED $(n=50)$ and transforaminal endoscopic discectomy (PTED; n=36) in our hospital. All patients were followed-up with self-evaluation questionnaires, Oswestry disability index (ODI), medical outcomes study 36-item short form health survey and MacNab criteria. All the patients in both groups were followed up to 12 months after the operation.

Results: ODI questionnaire responses were not statistically different between the MED and PTED groups (53.00 vs. 48.72) before treatment. Average scores and minimal disability after 5 days to 12 months of follow-up were 4.96 in the MED group and 3.61 in the PTED group. According to MacNab criteria, $92.0 \%$ of the MED group and $94.4 \%$ of the PTED group had excellent or good results with no significant difference.

Conclusions: There was no significant difference between MED and PTED outcomes. Further large-scale, randomized studies with long-term follow-up are needed.
\end{abstract}

Keywords: Intervertebral disc degeneration; Lumber disc herniation; Endoscopic discectomy; Minimally invasive surgery

\section{Introduction}

Lumbar disc herniation (LDH) is the most common cause of lower back pain and sciatica. The number of patients with LDH is increasing in all population including chil- dren. About $70 \%-85 \%$ of people suffer at least one episode of lower back pain with or without leg pain during their lives, which is the most common reason for hospital visits [1]. For patients who do not achieve good recovery with conservative treatment, surgical intervention should

Received Mar 17, 2015; Revised Apr 9, 2015; Accepted Apr 9, 2015

Corresponding author: Xiao-Tao $\mathrm{Wu}$

Department of Spine Surgery, Zhongda Hospital, Southeast University,

No.87, Dingjiaqiao Road, Nanjing, China, 210009

Tel: +86-25-83262334, Fax: +86-25-83262331, E-mail: wuxiaotao@medmail.com.cn 
be considered. As a result, numerous surgical interventions are performed. These include microendoscopic discectomy (MED), hemilaminectomy with discectomy, chemonucleolysis, nucleoplasty, percutaneous laser discectomy and percutaneous transforaminal endoscopic discectomy (PTED). Endoscopic techniques are being refined to permit discectomy under direct view and local anesthesia.

Studies in the United States [2] and Japan [3] over 40 years ago explored non-visualized percutaneous central nucleotomy for the resection and elimination of the tissue through a posterior approach. PTED indications are the same as classical discectomy procedures [4]. The entrance route is intramuscular in which epidural and neural vein is protected. Connective tissues, lagamentum flavum, and posterior longitudinal ligaments are also protected. Due to its low level of invasiveness, postoperative back pain is minimal and, therefore, patients derive satisfaction because of fast recovery. PTED is an effective method for recurrent LDH with less complications and high patient satisfaction [5]. PTED is often used in Korea [6,7] Germany $[8,9]$ and the United States [10].

MED was first introduced in 1997 as a minimally invasive surgical approach using advanced optics [11]. Symptomatic nerve root decompression is the main purpose of MED. The perceived benefits are less muscle and soft tissue damage with better visualization. MED is an impressive surgical technique with good long-term outcomes in the treatment of LDH. It allows smaller incisions and less tissue trauma comparing to standard open microdiscectomy [12].

\section{Materials and Methods}

\section{Data collection}

The ethics committee of the medical school of Southeast University approved this study protocol. The data of the consecutive hospitalized patients with LDH treated with MED ( $\mathrm{n}=90)$ and PTED ( $\mathrm{n}=80)$ in Zhongda hospital between June 2013 and February 2014 were collected. Among these patients, 50 patients in the MED group and 36 patients in the PTED group were suitable for our study. The demographic characteristics of the patients including age, sex, duration of lower back pain, leg pain, segment of lower disc herniation and straight leg raising test were recorded. Quality of life measures (Oswestry disability index, ODI), medical outcomes study 36 -item shortterm health survey (SF-36) and the outcomes of MacNab's response were collected 5 days after operation during hospitalization and 3 months, 6 months and 12 months post-discharge by phone interviews. Magnetic resonance imaging (MRI) and computed tomography (CT) scans were performed in all cases.

\section{Inclusion and exclusion criteria}

The same inclusion and exclusion criteria were defined in both groups. Inclusion criteria were: (1) contained disc protrusion on preoperative MRI and CT scans; (2) neurological signs including motor weakness, sensory changes, radiculopathy and the presence of abnormal reflex due to migrated discs; (3) unsuccessful conservative treatment for at least 6 weeks; (4) age of 30-55 years at time of procedure and (5) no previous lumbar surgery on the same disc level. Exclusion criteria were: (1) narrowing foramen; (2) central spinal canal stenosis or lateral recess stenosis; (3) sequestered disc below or above the center of the pedicle of the lower vertebral body; (4) coexisting somatic or psychological condition, such as diabetes, cardiovascular diseases, spinal tumor or fracture, infection and (5) patient unreachable because of lack of correspondence address.

\section{Surgical techniques}

\section{1) Microendoscopic discectomy}

Surgery was carried out under spinal or general anesthesia with the patient in the prone position, with the abdomen free to reduce intraoperative venous bleeding on the Hall frame. A longitudinal skin incision of $16-20 \mathrm{~mm}$ was made approximately $3 \mathrm{~cm}$ lateral to the midline. The METRx MED system (Medtronic Sofamor Danek lnc., Memphis, TN, USA) was used for surgery. After dissection of the fascia, a diameter of $5.3 \mathrm{~mm}$ was inserted toward the inter lamina spaces, under the guidance of $\mathrm{C}$-arm fluoroscopic and the anatomic marker of the inferior border of the upper vertebral lamina. Dilators with larger diameters were inserted sequentially, and a tubular retractor with a diameter of $16 \mathrm{~mm}$ was finally put in place, with the tube tip contacting the inferior border of the cranial lamina and the medial border of the inferior articlular process. To fully expose the ligamentum flavum, the adhering soft tissues together with part of the bony structures from 
the lamina and the articlualr process were removed. After the ligamentum flavum was split longitudinally, the compressed dural sac and nerve root were exposed and tracked contralaterally to localize the herniated disc tissues, which were removed carefully to decompress the nerve root. Following discectomy, the intervertebral space was washed with saline solution to swill out the remaining fragments. Suction drainage was placed in the surgical wound and removed after bleeding decreased (the first postoperative day in most cases).

\section{2) Percutaneous transforaminal endoscopic discectomy} Surgery was carried out with the patient in the prone position under local anesthesia, with the back mildly flexed. The optimal skin entry point was more lateral $(8-14 \mathrm{~cm}$ from the midline). An 18 gauge spinal needle was gently introduced laterally under fluoroscopic guidance, through a triangular working zone into the intervertebral disc until it contacted the annular surface. This zone was formed (on lateral view) posteriorly by the superior facet joint, inferiorly by the upper endplate of the caudal vertebrae, and superiorly and anteriorly by the nerve root exiting the neural foramen. The superior facet joint was used as anatomic marker to avoid puncturing injuries and compressions to the existing nerve root. After the spinal needle punctured into the targeted disc, a mixture of contrast media and indigo carmine was introduced to stain the pathological nucleus and estimate the location of the annular tear. The spinal needle was then replaced with guide wire, through which the working channel was placed in order from thin to wide dilators. After the dilators passed through the annulus fibrosus, a beveled working cannula was slid over the dilator and the guide wire, followed by the connection to the endoscope and irrigation system. The herniated and degenerated disc tissues were identified by the blue staining with the indigo carmine, which were removed till the posterior longitudinal ligament was loosened and the pulse of the dura sac recovered. The wound was closed without draining and the patient was sent back to the ward for further observation at least 2 days before discharge.

\section{Clinical evaluations}

Self-evaluation questionnaires of the ODI, short form 36 (SF-36) and MacNab criteria were used as indices of clinical outcomes. This evaluation also included recurrence of symptoms, complications, subsequent surgical therapy, duration of hospitalization, length of operative time and average expenses, all of which were reviewed by telephone calls and initial records of the patients in Zhongda Hospital. In this study, the average expenses included the costs of diagnosis, surgery, medication and the treatment during hospitalization.

1) Oswestry disability index

ODI is divided into 10 items aimed to assess multiple aspects of disability with respect to pain: pain intensity, personal care, lifting, walking, sitting, standing, sleeping, sex life, social life and travelling. The sex life question (section 8) is unacceptable for cultural reasons in China. Therefore, it was removed from the questionnaire. The total possible score became 45 . The final score is calculated and presented as a percentage ( $0 \%$ represents no pain and disability and $100 \%$ represents the worst possible pain and disability).

\section{2) 36-Item short form health survey}

The SF-36 health survey questionnaire is typically used to assess health-related quality of life. The questionnaire is comprised of 36 items on physical and social status of the patient divided in eight groups: (1) PF, physical functioning; (2) RP, role limitations due to physical health; (3) BP, body pain; (4) GH, general health; (5) VT, energy/fatigue; (6) SF, social functioning; (7) RE, role emotional; and (8) $\mathrm{MH}$, mental health. The questions are scored on a scale of 0 (worst health) to 100 (ideal health).

\section{3) MacNab criteria}

The MacNab criteria defined excellent outcome as no pain and no restriction of activity; good outcome as occasional pain, but no need of medication and no restriction of activity; fair outcome as somewhat improved and need of medication, with some restriction of daily activity and poor outcome as no improvement or worsening, and/or need for further operative intervention.

\section{Statistical analysis}

Data were input in a Microsoft Excel database, and analysis was performed by IBM SPSS ver. 20.0 (SPSS Inc., Chicago, IL, USA). Comparison between subgroups was made with rank sum test, paired-sample $t$-tests, Fisher exact tests and a $p<0.05$ was considered statistically significant. 
Table 1. Demographics

\begin{tabular}{|c|c|c|c|}
\hline Item & $\operatorname{MED}(n=50)$ & PTED $(n=36)$ & $p$-value \\
\hline Age (yr) & & & 0.078 \\
\hline MeanıSD (range) & $41.46 \pm 7.22(30-55)$ & $44.17 \pm 6.54(30-55)$ & \\
\hline $\operatorname{Sex}(n)$ & & & 0.582 \\
\hline Male & $29(55.8)$ & $23(44.2)$ & \\
\hline Female & $21(61.8)$ & $13(38.2)$ & \\
\hline \multicolumn{4}{|c|}{ Duration of lower back pain (n) } \\
\hline$\leq 3 \mathrm{mo}$ & $11(22.9)$ & $16(44.4)$ & 0.028 \\
\hline $3 \mathrm{mo}$ to $3 \mathrm{yr}$ & $19(38.0)$ & $14(38.9)$ & \\
\hline$\geq 3 \mathrm{yr}$ & $20(40.0)$ & $6(16.7)$ & \\
\hline \multicolumn{4}{|l|}{ Duration of leg pain (n) } \\
\hline$\leq 3 \mathrm{mo}$ & $23(46.0)$ & $15(41.7)$ & 0.803 \\
\hline $3 \mathrm{mo}$ to $3 \mathrm{yr}$ & $16(32.0)$ & $14(38.9)$ & \\
\hline$\geq 3 \mathrm{yr}$ & $11(22.0)$ & $7(19.4)$ & \\
\hline Straight leg raising test ( $\mathrm{n}$ ) & & & 0.160 \\
\hline$\leq 40$ degrees & $24(48.0)$ & $10(27.8)$ & \\
\hline 40-70 degrees & $12(24.0)$ & $11(30.6)$ & \\
\hline$\geq 70$ degrees & $14(28.0)$ & $15(41.7)$ & \\
\hline Disk level treated (n) & & & $0.005^{a)}$ \\
\hline $\mathrm{L} 3 / 4$ & $1(2.0)$ & $3(8.3)$ & \\
\hline$L 3 / 4 L 4 / 5$ & $0(0.0)$ & $4(11.1)$ & \\
\hline L3/4 L4/5 L5/S1 & $2(4.0)$ & $0(0.0)$ & \\
\hline$\llcorner 4 / 5$ & $17(34.0)$ & $13(36.1)$ & \\
\hline L4/5 L5/S1 & $8(16.0)$ & 0 & \\
\hline L5/S1 & $22(44.0)$ & 16 (44.4) & \\
\hline
\end{tabular}

MED, microendoscopic discectomy; PTED, percutaneous tranforaminal endoscopic discectomy; SD, standard deviation.

a) Fisher's exact test.

\section{Results}

\section{Demographic data}

Demographic data are summarized in Table 1. The series consisted of 29 males and 21 females (total 50 patients) in the MED group, and 23 males and 13 females (total 36 patients) in the PTED group. The mean age at the time of operation was $41.46 \pm 7.22$ years in the MED group and $44.17 \pm 6.54$ years in the PTED group (range, 30 to 55 years in both groups).

\section{Collective objective results of MED and PTED groups}

There was a significant difference between the mean op- erative time of the MED group (46.90 \pm 14.74 minutes) and the PTED group $(93.89 \pm 32.33$ minutes; $p<0.001)$ (Table 2). The mean average length of hospital stay in the MED group (5.54 \pm 1.72 days) and in the PTED group $(5.05 \pm 2.20$ days) was similar, with no significant difference $(p<0.255)$. Comparison of the average expenses of the MED and PTED groups revealed significantly lower cost in the MED group $(p<0.001)$. There were no serious complications in either groups including cauda equine syndrome, nerve root injury, spondylodiscitis or thrombosis.

\section{Oswestry disability index}

Preoperative ODI scores and the postoperative scores at 
Table 2. Collective objectives results of MED and PTED groups

\begin{tabular}{|c|c|c|}
\hline Objective outcomes measures & MED & PTED \\
\hline Operation time $(\min )^{a)}$ & $46.90 \pm 14.74$ & $93.89 \pm 32.33$ \\
\hline Average length of hospital stay (day) & $5.54 \pm 1.72$ & $5.05 \pm 2.20$ \\
\hline Average expenses $(\$)^{c)}$ & $1,700 \pm 470$ & $2,480 \pm 450$ \\
\hline
\end{tabular}

MED, microendoscopic discectomy; PTED, percutaneous tranforaminal endoscopic discectomy.

${ }^{\text {al }}$ T test, $t=-8.131, p<0.001$; ${ }^{\text {bl}}$ T test, $t=1.146, p<0.255$; ${ }^{\mathrm{c}} \mathrm{T}$ test, $t=-7.720, p<0.001$.

Table 3. ODI scores of patients in the MED and PTED groups

\begin{tabular}{|c|c|c|c|c|c|c|}
\hline \multirow{2}{*}{ Group } & \multicolumn{5}{|c|}{ ODI } & \multirow{2}{*}{$\begin{array}{l}\text { Average score } \\
\text { (mean } \pm S D)\end{array}$} \\
\hline & 0 & $1-8$ & $9-17$ & $18-26$ & $>26$ & \\
\hline \multicolumn{7}{|l|}{ MED group } \\
\hline Before treatment ${ }^{a)}$ & 0 & 0 & 0 & 1 & 49 & $53.00 \pm 14.18$ \\
\hline After treatment ${ }^{\mathrm{b})}$ & 34 & 6 & 3 & 5 & 2 & $4.96 \pm 10.34$ \\
\hline \multicolumn{7}{|l|}{ PTED group } \\
\hline Before treatment & 0 & 0 & 0 & 1 & 35 & $48.72 \pm 11.55$ \\
\hline After treatment & 26 & 5 & 1 & 3 & 1 & $3.61 \pm 8.5$ \\
\hline
\end{tabular}

ODI, Oswestry disability index; MED, microendoscopic discectomy; PTED, percutaneous transforaminal endoscopic discectomy; SD, standard deviation.

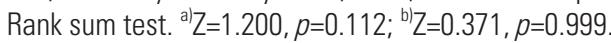

Table 4. SF-36 scores of patients in the MED and PTED groups

\begin{tabular}{lcccccccccc} 
Group & PF & RP & BP & GH & VT & SF & RE & MH & Total \\
MED group & 85.2 & 91.5 & 87.3 & 84.1 & 79.8 & 89.5 & 99.3 & 83.8 & 87.57 \\
PTED group & 90.0 & 91.7 & 92.8 & 83.1 & 79.6 & 94.4 & 100.0 & 82.4 & 89.26 \\
$t$ & 2.450 & 0.061 & 1.720 & 0.738 & 0.285 & 2.456 & 0.847 & 1.112 & 1.407 \\
$p$-value & 0.016 & 0.952 & 0.089 & 0.463 & 0.776 & 0.016 & 0.399 & 0.269 & 0.163 \\
\hline
\end{tabular}

SF-36, short form 36; MED, microendoscopic discectomy; PTED, percutaneous transforaminal endoscopic discectomy; PF, physical functioning; RP, role limitations due to physical health; BP, body pain; $\mathrm{GH}$, general health; VT, energy/fatigue; SF, social functioning; RE, role emotional; MH, mental health.

12-month follow-up in the MED and PTED groups are shown in Table 3. Preoperative and postoperative scores between the groups were similar ( $p=0.112$ and $p=0.999$, respectively; rank sum test). In the MED group, no disability (ODI, 0 ) at the 1-year follow-up was found in 34 patients $(68 \%)$, minimal disability $(\mathrm{ODI}<20 \%)$ in 14 patients $(28 \%)$ and severe disability (ODI $>40 \%)$ in 2 patients (4\%). The respective values in the PTED group were 26 patients (72.23\%), 9 patients (25\%) and 1 patient (2.77\%).

\section{36-Item short form health survey}

SF-36 scores of the MED and PTED groups are presented in Table 4. Total average scores of the groups was not statistically significant $(p=0.163)$. PF and SF in the PTED group were significantly better than in MED group ( $p=0.016$ and $p=0.016$, respectively).

\section{MacNab Criteria}

For the MED group, 33 patients (66\%) had excellent results, 13 patients (26\%) good results and 4 patients (8\%) fair results. In the PTED group, 26 patients (72.2\%) had excellent results, 8 patients (22.2\%) good results, and 2 patients (5.6\%) fair results. Most of the patients in both groups (92.0\% in the MED group and $94.4 \%$ in the PTED group) 
Table 5. MacNab Scores of patients in the MED and PTED groups

\begin{tabular}{lcccc} 
Group & Excellent & Good & Fair & Total \\
MED & $33(66.0)$ & $13(26.0)$ & $4(8.0)$ & 50 \\
PTED & $26(72.2)$ & $8(22.2)$ & $2(5.6)$ & 36 \\
Total & 59 & 21 & 6 & 86 \\
\hline
\end{tabular}

Values are presented as number (\%).

Rank sum test. Z $=0.285, p=0.99$.

MED, microendoscopic discectomy; PTED, percutaneous transforaminal endoscopic discectomy.

achieved excellent or good results with no significant difference ( $p=0.99$ ) (Table 5).

\section{Discussion}

LDH is usually due to age related degeneration of the nucleus pulposus and annulus fibrosus, although straining, lifting injuries or trauma can be other causes [13]. Bulging out of the fibrous ring and nucleus pulposus from the intervertebral disc is one of the major causes of lower back pain. It is followed by intense pain around the affected disc as well as pain that radiates to the lower back and legs. Minimally invasive surgery has attracted growing attention because of the reduction in postoperative pain, earlier mobilization and shorter recovery.

MED was introduced in 1997 as a minimally invasive surgical approach using advanced optics [11]. The indications for this procedure are posterolateral disc herniation with or without lateral recess stenosis and foraminal and extraforaminal disc herniation. However, some spine surgeons reserve the MED procedure for small disc herniation. Some surgeons do not prefer to perform MED procedure because of difficulty to decompress the nerve root with a restricted surgical field of $18 \mathrm{~mm}$ diameter where working space is limited [12]. Schick et al. [14] conducted an intraoperative study of electromyographic activity, in which 15 patients were treated through an endoscopic technique and 15 patients through the open microscopic surgical technique for LDH. Results showed that the endoscopic technique was better than the open surgical technique, with less irritation of the nerve root. Complications of instability and nerve root injuries can be minimized by removing fewer spinal canal structures. Preservation of ligamentum flavum reduces epidural scarring and adhesions, which result in more difficult and timeconsuming reoperations [8]. Most (91\%-100\%) outcomes are excellent following MED $[11,15,16]$. In one study, 873 consecutive patients with LDH treated by MED achieved a significant improvement of ODI scores after treatment [12]. The authors stated that aged patients with segmental instability and patients with previous back surgery were not perfect candidates for MED. Knowing the impact of selection criteria on the outcome, we included patients 30 to 55 years of age.

Since 1963, less invasive decompressive procedures for herniated disc have been developed. These include chymopapain [17] and, about a decade later, percutaneous nucleotomy [3]. Lumbar microdiscectomy was introduced in 1979 and subsequently refined $[2,18,19]$. Percutaneous laser-nucleolyzers were introduced for the decompression of LDH in 1987 [20]. In 1994, a new instrument enabling enlargement of the foramen with special reamers was introduced, which made the anterior spinal canal accessible for endoscope and other instruments [5]. Recurrent herniated discs treated using endoscopic transforaminal discectomy achieves excellent or good results in about $85 \%$ of cases, with fewer complications [5]. The transforaminal approach reduces the traumatization and herniated discs can be removed sufficiently inside and outside the spinal canal [8].

Patients with shorter symptom durations ( $<6$ months) have a better outcome. This indicates that a recent soft herniation is easier to remove using an endoscope. Many studies have proven the safety and effectiveness of both MED and PTED [5,12,21-23], but there have been few studies of long-term follow-up for these two procedures. ODI is one of the specific outcome measures used in the management of spinal disorders and has become the gold standard outcome criterion. In this study, we compared preoperative and postoperative self-evaluation scores in MED and PTED groups. Some patients in our study were satisfied compared to their preoperative condition even 
though their ODI was less than 15 . Others were not happy despite an ODI score ranging between $0-8$, which could be due to factors like high cost or a final outcome that did not meet their expectation. These findings were similar to other studies.

The SF-36 health survey questionnaire has proved to be a valid and valuable health survey formats. It has been extensively used. SF-36 achieves the best balance between duration, dependability, cogency, reactivity and experience, even in large populations of patients with lower back pain. In the current study, physical and social functioning in the PTED group were significantly better than that in MED group (both $p=0.016$ ). The MacNab criterion is also a useful criterion for the assessment of patients with lower back pain. In our study, excellent and good results of $92.0 \%$ in the MED group and $94.4 \%$ in PTED group are obtained at 12 months postoperative follow-up.

Keeping in mind the amount and type of intervention required in both groups, the average length of hospital stays post-MED and post-PTED range between 1 and 2 days in western societies $[8,22]$. Presently, the mean average length of hospital stay in the MED group $(5.54 \pm 1.72$ days) and PTED group (5.05 \pm 2.20 days) was similar, possibly because patients were reluctant to leave the hospital before complete recovery and ample rehabilitation, even though the hospital staying charge was high. This finding was similar to another study [23], in which showed patients were usually discharged within 24 hours in the absence of complications.

Average expenses were significantly lower cost in the MED group $(p<0.001)$. Patients suffering from LDH prefer the PTED surgical technique even though this technique is more expensive compared with MED technique, because they have medical insurance and/or because of the additional many benefits of this procedure to the patients, such as local anesthesia and early off-bed activity. In contrast to a prior study [24], our study observed very limited complications. The complications in the prior study were occasional pain, failure of surgery and neurologic deterioration, which were not found in our study.

There are several limitations to this study. Firstly, because some patients with good results may refuse to come back for further examinations, nearly $50 \%$ of the operated patients (40 in the 90 MED group and 46 in the 80 PTED group) were not recruited into our study, thus a further correlative analysis was impossible based on the available data. Secondly, due to the nature of the retrospective observation and the lack of standardized indication for MED and PTED procedures, bias from the spine surgeons in patient selection might contribute to the un-significant difference between the MED and PTED outcomes. Therefore, further large-scale, randomized studies with longterm follow-up are needed.

\section{Conclusions}

Treatment of LDH by MED and PTED seeks optimal decompression of nerve roots, minimal tissue trauma and complications. Our study demonstrates satisfactory outcomes following MED and PTED, reflecting the advantages of being able to treat using a smaller incision, better pain relief and less chance of damage to the neural root and its tributaries. Further large-scale studies should be performed to evaluate the differences between these two surgical procedures.

\section{Conflict of Interest}

This work was supported by the Science and Technology Bureau of Jiangsu province (No.BL201330166). No potential conflict of interest relevant to this article was reported.

\section{References}

1. Andersson GB. Epidemiological features of chronic low-back pain. Lancet 1999;354:581-5.

2. Kambin P, Gellman H. Percutaneous lateral discectomy of the lumbar spine a preliminary report. Clin Orthop Relat Res 1983;174:127-32.

3. Hijikata S. Percutaneous nucleotomy: a new concept technique and 12 years' experience. Clin Orthop Relat Res 1989;(238):9-23.

4. Choi G, Lee SH, Bhanot A, Raiturker PP, Chae YS. Percutaneous endoscopic discectomy for extraforaminal lumbar disc herniations: extraforaminal targeted fragmentectomy technique using working channel endoscope. Spine (Phila Pa 1976) 2007;32:E93-9.

5. Hoogland T, van den Brekel-Dijkstra K, Schubert M, Miklitz B. Endoscopic transforaminal discectomy for recurrent lumbar disc herniation: a prospective, cohort evaluation of 262 consecutive cases. Spine (Phila Pa 1976) 2008;33:973-8.

6. Lee S, Kim SK, Lee SH, et al. Percutaneous endo- 
scopic lumbar discectomy for migrated disc herniation: classification of disc migration and surgical approaches. Eur Spine J 2007;16:431-7.

7. Choi G, Lee SH, Lokhande P, et al. Percutaneous endoscopic approach for highly migrated intracanal disc herniations by foraminoplastic technique using rigid working channel endoscope. Spine (Phila Pa 1976) 2008;33:E508-15.

8. Ruetten S, Komp M, Merk H, Godolias G. Fullendoscopic interlaminar and transforaminal lumbar discectomy versus conventional microsurgical technique: a prospective, randomized, controlled study. Spine (Phila Pa 1976) 2008;33:931-9.

9. Ruetten S, Komp M, Merk H, Godolias G. Fullendoscopic cervical posterior foraminotomy for the operation of lateral disc herniations using 5.9- $\mathrm{mm}$ endoscopes: a prospective, randomized, controlled study. Spine (Phila Pa 1976) 2008;33:940-8.

10. Hermantin FU, Peters T, Quartararo L, Kambin P. A prospective, randomized study comparing the results of open discectomy with those of video-assisted arthroscopic microdiscectomy. J Bone Joint Surg Am 1999;81:958-65.

11. Perez-Cruet MJ, Foley KT, Isaacs RE, et al. Microendoscopic lumbar discectomy: technical note. Neurosurgery 2002;51(5 Suppl):S129-36.

12. Wu X, Zhuang S, Mao Z, Chen H. Microendoscopic discectomy for lumbar disc herniation: surgical technique and outcome in 873 consecutive cases. Spine (Phila Pa 1976) 2006;31:2689-94.

13. Olmarker K, Rydevik B. Pathophysiology of sciatica. Orthop Clin North Am 1991;22:223-34.

14. Schick U, Dohnert J, Richter A, Konig A, Vitzthum H. Microendoscopic lumbar discectomy versus open surgery: an intraoperative EMG study. Eur Spine J 2002;11:20-6.

15. Foley KT, Smith MM, Rampersaud YR. Microendo- scopic approach to far-lateral lumbar disc herniation. Neurosurg Focus 1999;7:e5.

16. Chang SS, Fu TS, Liang YC, et al. Results of microendoscopic discectomy performed in the 26 cases with a minimum 3 years follow-up. Chang Gung Med J 2009;32:89-97.

17. Smith L, Garvin PJ, Gesler RM, Jennings RB. Enzyme dissolution of the nucleus pulposus. Nature 1963; 198:1311-2.

18. Kambin P, Casey K, O'Brien E, Zhou L. Transforaminal arthroscopic decompression of lateral recess stenosis. J Neurosurg 1996;84:462-7.

19. Kambin P, Gennarelli T, Hermantin F. Minimally invasive techniques in spinal surgery: current practice. Neurosurg Focus 1998;4:e8.

20. Choy DS. Risks of laser discolysis. J Neurosurg 1992; 77:978.

21. Ahn Y, Lee SH, Park WM, Lee HY, Shin SW, Kang HY. Percutaneous endoscopic lumbar discectomy for recurrent disc herniation: surgical technique, outcome, and prognostic factors of 43 consecutive cases. Spine (Phila Pa 1976) 2004;29:E326-32.

22. Schizas C, Tsiridis E, Saksena J. Microendoscopic discectomy compared with standard microsurgical discectomy for treatment of uncontained or large contained disc herniations. Neurosurgery 2005;57:35760.

23. Ahn Y, Lee SH, Lee JH, Kim JU, Liu WC. Transforaminal percutaneous endoscopic lumbar discectomy for upper lumbar disc herniation: clinical outcome, prognostic factors, and technical consideration. Acta Neurochir (Wien) 2009;151:199-206.

24. Sencer A, Yorukoglu AG, Akcakaya MO, et al. Fully endoscopic interlaminar and transforaminal lumbar discectomy: short-term clinical results of 163 surgically treated patients. World Neurosurg 2014;82:88490. 